\title{
COMMUTATORS AND NORMAL OPERATORS
}

\author{
by M. J. CRABB and P. G. SPAIN
}

(Received 20 April, 1976)

Let $X$ be a Banach space and $L(X)$ the Banach algebra of bounded linear operators on $X$. An operator $T$ in $L(X)$ is hermitian if $\left\|e^{i t T}\right\|=1(t \in R)$, and is normal if $T=R+i J$ where $R$ and $J$ are commuting normal operators; $R$ and $J$ are then determined uniquely by $T$, and we may write $T^{*}=R-i J$. These definitions extend those for operators on Hilbert spaces. More details may be found in [1].

Given $T$ in $L(X)$ we may define the left-multiplication operator $\lambda_{T}: L(X) \rightarrow L(X)$ : $A \mapsto T A$ and the right-multiplication operator $\rho_{T}: L(X) \rightarrow L(X): A \rightarrow A T$. It is easy to check (see [2], for instance) that $\lambda_{T}$ and $\rho_{T}$ are hermitian in $L(L(X))$ if $T$ is hermitian in $L(X)$. It follows that $\lambda_{N_{1}}-\rho_{N_{2}}$ is normal in $L(L(X))$ if $N_{1}$ and $N_{2}$ are normal in $L(X)$.

Putnam [4] proved that if $H$ is a Hilbert space, if $A, B \in L(H)$, and if $A$ is normal and commutes with $A B-B A$, then $A$ commutes with $B$. The following result extends Putnam's theorem to operators on Banach spaces.

Proposition 1. Suppose $T=N+Q$ where $Q$ is quasinilpotent in $L(X), N=R+i J$ is normal in $L(X)$ and $N$ commutes with $Q$. Suppose further that $T^{2} x=0$ for some $x \in X$. Then $R x=J x=0$.

Proof. We first observe that $Q$ commutes with both $R$ and $J$ ([2], Lemma 3).

Let $Y=\overline{\operatorname{lin}}\left\{R^{p} J^{q} Q^{r} x: p, q, r=0,1,2, \ldots\right\}$. Then $Y$ is invariant under $R, J$ and $Q$; and $(T \mid Y)^{2}=0$. Thus $T|Y-Q| Y=R|Y+i J| Y$ is both normal and quasinilpotent. Hence (see [1], §38) $R|Y=J| Y=0$ : that is, $R x=J x=0$.

L. A. Harris has proved that if $N$ is normal in $L(X)$ and $N x=0$, then $N^{*} x=0$. This is an immediate corollary of the above proposition: for $N x=0$ implies $N^{2} x=0$, from which $R x=J x=0$. However, we do not always have $\|N x\|=\left\|N^{*} x\right\|$. For the operator $\lambda_{N_{1}}-\rho_{N_{2}}$ is normal on $L(H)$ when $H=\mathbf{C}^{2}, N_{1}=\operatorname{diag}(1,-1)$ and $N_{2}=\operatorname{diag}(i, 0)$; but $\left\|N_{1} T-T N_{2}\right\|=4$ while $\left\|N_{1}^{*} T-T N_{2}^{*}\right\|=2 \sqrt{2}$ if $T=\left[\begin{array}{rr}1+i & 2 \\ -1+i & -2\end{array}\right]$.

We give a short proof of the following result due to Palmer ([3], Lemma 2.7); the notation is as above.

Proposition 2. Let $N=R+i J$, where $R$ and $J$ commute and $R^{p} J^{q}$ is hermitian for $p, q=$ $0,1,2, \ldots ;$ then $\|N x\|=\left\|N^{*} x\right\|(x \in X)$.

Proof. The closure of the set of polynomials in $R$ and $J$ forms a commutative $C^{*}$-algebra under the operator norm and the natural involution (by the Vidav-Palmer theorem; see [1], chapter 5). For $\varepsilon>0$ the functional calculus gives

$$
\left\|N-N^{2}\left(N^{*} N+\varepsilon I\right)^{-1} N^{*}\right\|=\left\|\varepsilon N\left(N^{*} N+\varepsilon I\right)^{-1}\right\| \leqq \sqrt{\varepsilon} / 2,
$$

Glasgow Math. J. 18 (1977) 197-198. 
and $\left\|N^{2}\left(N^{*} N+\varepsilon I\right)^{-1}\right\| \leqq 1$. Hence, for $x \in X$,

$$
\|N x\|=\lim _{\varepsilon \rightarrow 0}\left\|N^{2}\left(N^{*} N+\varepsilon I\right)^{-1} N^{*} x\right\| \leqq\left\|N^{*} x\right\| .
$$

This gives $\left\|N^{*} x\right\| \leqq\left\|N^{* *} x\right\|=\|N x\|$, which completes the proof.

\section{REFERENCES}

1. F. F. Bonsall and J. Duncan, Complete normed algebras (Springer-Verlag, 1973).

2. H. R. Dowson, T. A. Gillespie and P. G. Spain, A commutativity theorem for hermitian operators, Math. Ann. 220 (1976), 215-217.

3. T. W. Palmer, Unbounded normal operators on Banach spaces, Trans. Amer. Math. Soc. 133 (1968), 385-414.

4. C. R. Putnam, On normal operators in Hilbert space, Amer. J. Math. 73 (1951), 357-362.

\section{UNIVERSITY OF GLASGOW}

GLASGOW 